\title{
SISTEMA DE PENSIONES EN EL PERÚ: LA SIGUIENTE REFORMA
}

\section{RESUMEN}

En este artículo se propone una reforma del sistema de pensiones en el Perú que consiste crear una Administradora de Fondos de Pensiones, pública y autónoma, que realice las tareas del actual Sistema Nacional de Pensiones y el Sistema Privado de Pensiones. Mediante esta reforma se podría ganar mayor eficiencia a través de las economías de escala y mejorar la asignación de recursos tanto del Estado como de los aportantes a ambos sistemas.

JEL: G23

PALABRA CLAVE: Sistema de Pensiones, Reforma

\begin{abstract}
This study proposes a reform of the pension system in Peru that consists of creating a Public and Autonomous Pension Funds Administrator that performs the tasks of the current National Pension System and the Private Pension System. Through this reform, greater efficiency could be gained through economies of scale and improve the allocation of resources by both, the State and the contributors to both systems.
\end{abstract}

JEL: G23

KEY WORD: Pension System, Reform

\footnotetext{
A Fredy Espino es Docente de la Escuela Profesional de Economía. Facultad de Ciencias Contables, Económicas y Financieras de la Universidad de San Martín de Porres (USMP). Las opiniones vertidas en este documento no necesariamente coinciden con las de la USMP ni con las de otras instituciones a las que pertenece el autor. El autor agradece los comentarios de Raúl Moquillaza a versiones anteriores que dieron origen al presente artículo.
} 


\section{INTRODUCCIÓN}

En este artículo se propone un reforma del sistema de pensiones en el Perú que consiste crear una Administradora de Fondos de Pensiones, pública y autónoma, de tal manera que realice las tareas del Sistema Nacional de Pensiones (SNP), bajo la Ley $\mathrm{N}^{\circ} 19990$, y el Sistema Privado de Pensiones (SPP), con la finalidad de ganar mayor eficiencia en la asignación de recursos. Para ello, se plantea el diseño de una comisión única en función al saldo de los fondos de pensión y de la rentabilidad de los mismos, a través de la cual hará sostenible la labor de administración de los fondos de pensiones.

Lograr un sistema de pensiones para la jubilación ideal es una tarea continua que tiene que adaptarse a las sociedades y a los contextos en los que ellas se desenvuelven. Por lo tanto, las reformas aplicadas a dicho objetivo no tienen respuesta única, tal como señalan Carranza et al. (2016). En el Perú, el sistema de pensiones actual es consecuencia de un proceso largo de reformas durante el Siglo $\mathrm{XX}$ y hoy cuenta con dos sistemas uno privado, el SPP, y otro público, el SNP, los cuales aún no logran alcanzar un nivel óptimo en la provisión de pensiones de jubilación, a un costo alto para los afiliados y el Estado.

La existencia de dos sistemas de pensiones no sólo genera distorsiones en los agentes sobre cuál sistema elegir, si no que provoca una mala asignación de los recursos tanto de los afiliados como del Estado. En el caso de los afiliados, la mala asignación se refleja en las altas comisiones que pagan en el SPP, sobre todo cuando ante eventos adversos del mercado los únicos perdedores son los afiliados y no el SPP. En el caso del Estado, el déficit de aportantes comparado con las pensiones que tiene que cubrir se va a ir ampliando en el largo plazo, incrementando el gasto del Estado en dicho rubro cuando debería destinar dichos fondos a otros temas prioritarios como educación, salud o justicia.

Por lo tanto, en este artículo nos preguntamos acerca de cuál es la siguiente reforma que se debe realizar para lograr un sistema de pensiones ideal. De esta manera se propone que la siguiente reforma del sistema de pensiones en el Perú consiste en la creación de una sola Administradora de Fondos de Pensiones pública y autónoma de tal manera que realice la tarea que vienen realizando los dos sistemas. Con la creación de esta entidad, se ganará eficiencia en la asignación de recursos tanto de los afiliados como del Estado, debido a las economías de escala. El marco regulatorio de esta nueva entidad sería sobre la base de la existente para el SPP.

El resto del artículo se organiza de la siguiente manera. En la segunda parte se analiza las características del SPP, enfocándose en las variables de decisión de los afiliados para escoger a las AFP, las comisiones y la rentabilidad de los fondos de pensión, y luego se analiza la estructura de costos de las AFP. En la tercera parte se plantea las recomendaciones de política. Finalmente, en la cuarta parte se concluye.

\section{EL SISTEMA DE PENSIONES EN EL PERÚ EN LA ACTUALIDAD}

En el Perú coexisten dos sistemas de pensiones: uno es el Sistema Nacional de Pensiones (SNP) conformada por la Oficina de Normalización Previsional $(\mathrm{ONP})^{1}$, y el otro es el Sistema Privado de Pensiones (SPP), conformado por las Administradoras Privadas de Fondos de Pensiones $(\mathrm{AFP})^{2}$. El primero, se basa en un esquema de reparto, esto es, los afiliados aportan a un fondo compartido del cual se derivará el pago de sus pensiones al momento de su jubilación, por lo que las generaciones jóvenes terminan financiando a las más longevas. El segundo, se basa en uno de fondo de cuentas individuales de capitalización (CIC), es decir, cada afiliado aporta a su propio fondo durante un periodo aproximado de 40 a 45 años el cual irá obteniendo rentabilidad para finalmente obtener su pensión de jubilación.

El SNP se creó durante el gobierno Juan Velasco en 1973 a través del Decreto Ley $N^{\circ} 19990$, el cual unificó la Caja Nacional de Seguro Social, el Seguro Social del Empleado y el Fondo Especial de Jubilación de los Empleados Particulares (Muñoz, 2000). Durante la década de 1980 presentó serios problemas financieros, por lo que hacía prever una reforma profunda en el sistema nacional de pensiones (Muñoz, 2000;

\footnotetext{
1 La ONP fue creada mediante el Decreto Ley $\mathrm{N}^{\circ}$ 25967, modificada por la Ley $\mathrm{N}^{\circ} 26323$ que le encargó, a partir del 1 de junio de 1994, la administración del SNP y del Fondo de Pensiones regulados por el Decreto Ley N ${ }^{\circ} 19990$. Adicionalmente se otorgó a la institución la gestión de otros regimenes pensionarios administrados por el Estado, como los de la Ley $\mathrm{N}^{\circ} 205030$.

2 El Sistema Privado de Pensiones (SPP) fue creado el 6 de diciembre de 1992, a través del Decreto Ley 25897. Está supervisado por la Superintendencia de Banca, Seguros y AFP (SBS).
} 
Cruz-Saco et al., 2014), lo cual originó la creación del SPP en forma paralela al SNP con la finalidad de aliviar la carga fiscal y proveer un medio más adecuado de ahorro para las pensiones de jubilación. En la actualidad los problemas de sostenibilidad persisten (MEF, 2004; CruzSaco et al., 2014), y las proyecciones realizadas hacia el año 2050 muestra al SNP insostenible desde el punto de vista financiero (Bernal et al., 2008). Por lo tanto, es fundamental plantear una reforma del SNP.

Luego de 19 años de creación del SNP, se creó el SPP durante el gobierno de Alberto Fujimori en 1992, Decreto Ley N² ${ }^{\circ} 5897$, como consecuencia de la pésima gestión del SNP durante la década de 1980, aunque no con el propósito de reemplazarlo en su totalidad, sino más bien como un sistema paralelo con la finalidad de hacer sostenible el sistema de pensiones. Durante su existencia, el SPP ha tenido efectos positivos en el ámbito macroeconómico, favoreciendo el incremento del ahorro y el dinamismo en el mercado de capitales (Bernal et al., 2008; CruzSaco et al., 2014; Alonso et al., 2014). Desde entonces se han realizado mejoras en el sistema, siendo la de mayor impacto la "Ley de Reforma del Sistema Privado de Pensiones" promulgada en 2012 (Ley $\mathrm{N}^{\circ}$ 29903). Los pilares principales de esta Ley comprenden mejoras en los tipos de fondos, las comisiones, la afiliación de trabajadores independientes, bases de licitación y participación de las AFP, etc. A pesar de esto, se sigue discutiendo que es lo que se debe hacer para mejorar dicho sistema, como la mejora en las comisiones, la incorporación de trabajadores independientes, las barreras a la entrada de nuevos competidores, etc. (Mendiola et al. 2013; Alonso et al., 2014).

De esta manera, tenemos dos sistemas que necesitan una mejora. En el caso del SNP es claro que es insostenible el esquema de reparto, por lo que todo debe apuntar hacia el esquema de las cuentas de capitalización individuales, tal como es el sistema privado. En el caso del SPP, el esquema oligopólico del mercado no genera la competencia esperada de tal manera que los afiliados vean menores comisiones en el largo plazo. En términos de eficiencia, la mejora de ambos sistemas se resume en una mejora del sistema de pensiones en general, luego de 45 y 26 años de creado el SNP y el SPP respectivamente. Por lo tanto, es pertinente resolver la pregunta sobre cuál es la siguiente reforma que se debe realizar en el sistema de pensiones en el Perú.
Debido a que el SNP ha sido largamente analizado, cuyas conclusiones confluyen hacia la misma idea de que es insostenible en el largo plazo, en la siguiente sección se analiza sólo el SPP desde el punto de vista del afiliado, con la finalidad de mostrar que la existencia de un esquema oligopólico en dicho sistema no es eficiente, para luego proponer las reformas en el sistema de pensiones.

\section{EL SPP DESDE EL PUNTO DE VISTA DEL AFILIADO}

El mercado del SPP es muy particular. La demanda de este mercado está conformada por los afiliados que tienen la obligación de aportar a dicho sistema. Por otro lado, la oferta está conformada por cuatro AFP, lo cual calza con un modelo de mercado oligopólico, cuya única fuente de ingreso son las comisiones que pagan los afiliados por la administración de sus fondos. Esto último hace posible la concertación de precios entre las AFP, razón por la cual las comisiones no bajan sustancialmente.

De esta manera, los afiliados sólo pueden elegir entre cuatro AFP, para lo cual observan dos variables principalmente: las comisiones y la rentabilidad de los fondos. Se asume que los afiliados deben escoger aquella AFP que le ofrece menores comisiones y mayor rentabilidad; sin embargo, eso no ocurre en la práctica, lo cual pasamos a analizar en los siguientes puntos.

\section{Rentabilidad de los fondos de pensión}

La rentabilidad de los fondos de pensión es una de las variables que los afiliados toman en cuenta para decidir en cuál AFP aportar. La Superintendencia de Banca, Seguros y AFP (SBS) publica la rentabilidad nominal y la real, a la cual se le descuenta la inflación. Entre ambas, la más comprensible para el afiliado promedio es la rentabilidad nominal de fin de periodo, que es la que indica cuánto ha ganado el fondo de pensiones en un determinado año.

En la Tabla 1 se muestra la rentabilidad nominal para todas las AFP durante el periodo 2008 2017 para los distintos fondos de pensión. En ella se observa dos características principales: uno, es que la rentabilidad de las AFP para los distintos fondos tienen una alta correlación, es decir, tienen evoluciones muy similares. La otra característica es que la discrepancia entre ellas es mínima, registrándose un rango 
máximo y mínimo de $[5.39 \%, 0.10 \%]$, [4.66\%, $1.06 \%]$ y $[5.88 \%, 1.52 \%]$ para los fondos 1,2 y 3 respectivamente. Es decir, tienen la misma evolución a niveles similares, en suma, no hay diferencia significativa entre escoger una $u$ otra AFP.

Tabla 1.

Rentabildiad Neta Nominal Anual (\%)

Fondo 1

\begin{tabular}{r|ccccc|ccc}
\hline & Habitat & Integra & Prima & Profuturo & Horizonte & Max & Min & Rango \\
\hline Dic-08 & & $-4,70$ & $-5,04$ & $-6,37$ & $-1,54$ & $-1,54$ & $-6,37$ & 4,83 \\
Dic-09 & & 14,19 & 15,64 & 16,84 & 19,58 & 19,58 & 14,19 & 5,39 \\
Dic-10 & & 5,69 & 8,90 & 7,09 & 9,90 & 9,90 & 5,69 & 4,22 \\
Dic-11 & & 2,19 & 3,30 & 2,05 & 4,21 & 4,21 & 2,05 & 2,16 \\
Dic-12 & & 12,95 & 12,04 & 11,35 & 12,88 & 12,95 & 11,35 & 1,60 \\
Dic-13 & & 0,00 & 0,11 & 0,24 & & 0,24 & 0,00 & 0,24 \\
Dic-14 & & 7,85 & 7,76 & 7,80 & & 7,85 & 7,76 & 0,10 \\
Dic-15 & & 6,26 & 4,27 & 6,12 & & 6,26 & 4,27 & 1,99 \\
Dic-16 & 10,54 & 7,92 & 8,64 & 6,52 & & 10,54 & 6,52 & 4,03 \\
Dic-17 & 10,64 & 9,33 & 9,60 & 8,60 & & 10,64 & 8,60 & 2,03 \\
\hline
\end{tabular}

Fondo 2

\begin{tabular}{r|ccccc|ccc}
\hline & Habitat & Integra & Prima & Profuturo & Horizonte & Max & Min & Rango \\
\hline Dic-08 & & $-21,47$ & $-21,09$ & $-21,38$ & $-23,53$ & $-21,09$ & $-23,53$ & 2,44 \\
Dic-09 & & 33,24 & 32,62 & 33,47 & 33,69 & 33,69 & 32,62 & 1,07 \\
Dic-10 & & 16,45 & 21,11 & 19,53 & 17,06 & 21,11 & 16,45 & 4,66 \\
Dic-11 & & $-6,87$ & $-7,13$ & $-5,93$ & $-7,92$ & $-5,93$ & $-7,92$ & 1,99 \\
Dic-12 & & 11,32 & 12,36 & 11,30 & 11,93 & 12,36 & 11,30 & 1,06 \\
Dic-13 & & 1,11 & $-2,43$ & 0,87 & & 1,11 & $-2,43$ & 3,54 \\
Dic-14 & & 8,86 & 9,61 & 8,23 & & 9,61 & 8,23 & 1,38 \\
Dic-15 & 7,43 & 5,39 & 4,91 & 6,32 & & 7,43 & 4,91 & 2,53 \\
Dic-16 & 11,01 & 10,32 & 10,18 & 9,10 & & 11,01 & 9,10 & 1,90 \\
Dic-17 & 13,80 & 12,57 & 12,16 & 10,65 & & 13,80 & 10,65 & 3,14 \\
\hline
\end{tabular}

Fondo 3

\begin{tabular}{r|ccccc|ccc}
\hline & Habitat & Integra & Prima & Profuturo & Horizonte & Max & Min & Rango \\
\hline Dic-08 & & $-39,70$ & $-38,44$ & $-37,26$ & $-34,57$ & $-34,57$ & $-39,70$ & 5,13 \\
Dic-09 & & 54,25 & 50,53 & 49,68 & 55,03 & 55,03 & 49,68 & 5,35 \\
Dic-10 & & 34,00 & 35,65 & 34,15 & 33,92 & 35,65 & 33,92 & 1,73 \\
Dic-11 & & $-16,33$ & $-19,83$ & $-16,27$ & $-15,91$ & $-15,91$ & $-19,83$ & 3,92 \\
Dic-12 & & 9,00 & 11,52 & 9,68 & 12,30 & 12,30 & 9,00 & 3,30 \\
Dic-13 & & 1,53 & $-2,78$ & 3,11 & & 3,11 & $-2,78$ & 5,88 \\
Dic-14 & & 6,09 & 7,61 & 6,27 & & 7,61 & 6,09 & 1,52 \\
Dic-15 & & 1,83 & 1,27 & 4,74 & & 4,74 & 1,27 & 3,47 \\
Dic-16 & 12,03 & 10,79 & 10,01 & 10,28 & & 12,03 & 10,01 & 2,02 \\
Dic-17 & 14,55 & 13,64 & 12,06 & 11,19 & & 14,55 & 11,19 & 3,35 \\
\hline
\end{tabular}

Fuente: SBS.

Elaboración: Propia.

Lo mostrado no es sorprendente, debido a que las inversiones de las AFP están reguladas por la SBS, por la que siguen un marco común, y sólo tienen grados de libertad en el activo particular que seleccionan en su cartera y no en el tipo de activo. Debido a ello, ocurre que la discrepancia que existe entre las rentabilidades de los fondos de pensión de las AFP va convergiendo o intercalando periodo a periodo.
Adicionalmente, es importante notar que la rentabilidad de los fondos de pensiones es una variable aleatoria, es decir, no se sabe el resultado hasta que ocurra el hecho. Aplicado al caso, los afiliados no saben cuál será la rentabilidad de sus fondos hasta que haya transcurrido el periodo de estimación, lo cual se explica porque los afiliados forman sus expectativas de rentabilidad de sus fondos de pensiones en función de la 
rentabilidad histórica, la cual no necesariamente tiene que ser igual a la que observarán. De esta manera, escoger entre una AFP $u$ otra debido a la rentabilidad es un argumento que se debilita, debido a que esta no está garantizada por la naturaleza misma de la variable.

Dada la similitud de la rentabilidad de los fondos de las AFP, tanto en evolución como en niveles, y dado el carácter aleatorio de estas, la rentabilidad pierde fuerza para ser considerada una variable de elección de los afiliados para escoger entre las AFP. Esto tiene impacto en la justificación para la existencia de cuatro AFP en el mercado actual.

En el siguiente punto se analiza las comisiones que pagan los afiliados por la administración de sus fondos, considerada como otra de las variables que ayudan a decidir entre las APF.

\section{Comisiones}

Las comisiones es el costo asumido por los afiliados por la administración de sus fondos de pensión. En la actualidad existen dos tipos de comisión: una sobre el flujo, equivalente a un porcentaje de la remuneración bruta de los afiliados, y la otra una comisión mixta, que se descompone en un porcentaje sobre el salario bruto más una comisión anual sobre el saldo del fondo de los afiliados.

En la Tabla 1 se muestra la comisión sobre el flujo, equivalente a un porcentaje de la remuneración bruta de los afiliados. El primer componente es la comisión sobre la remuneración bruta y el otro es la prima de seguro, y la suma de ambos es lo que denominamos comisión total. Al igual que la rentabilidad, destaca el hecho de que tienen evolución similar y tienen niveles similares, aunque a diferencia de la rentabilidad, la discrepancia entre las AFP es mínima. Enfocándonos en la comisión sobre el flujo total, que incluye la comisión sobre el salario y la prima por seguro, vemos que el rango para fines de 2017 es de $0.22 \%$, lo cual equivale a S/ 29.26 de ahorro anual para un afiliado cuyo salario es el mínimo de $\mathrm{S} / 950$, el cual es un monto extremadamente ínfimo como para incentivar al afiliado a cambiarse de una AFP debido sólo a las comisiones.

Tabla 2.

Comisión Sobre el Flujo

(\% Remuneración Bruta)

\begin{tabular}{r|ccccc|ccc}
\hline & Habitat & Integra & Prima & Profuturo & Horizonte & Max & Min & Rango \\
\hline Dic-08 & & 1,80 & 1,75 & 1,98 & 1,95 & 1,98 & 1,75 & 0,23 \\
Dic-09 & & 1,80 & 1,75 & 2,30 & 1,95 & 2,30 & 1,75 & 0,55 \\
Dic-10 & & 1,80 & 1,75 & 2,30 & 1,95 & 2,30 & 1,75 & 0,55 \\
Dic-11 & & 1,80 & 1,75 & 2,17 & 1,95 & 2,17 & 1,75 & 0,42 \\
Dic-12 & & 1,74 & 1,60 & 2,10 & 1,89 & 2,10 & 1,60 & 0,50 \\
Dic-13 & 1,47 & 1,55 & 1,60 & 1,84 & & 1,84 & 1,47 & 0,37 \\
Dic-14 & 1,47 & 1,55 & 1,60 & 1,69 & & 1,69 & 1,47 & 0,22 \\
Dic-15 & 1,47 & 1,55 & 1,60 & 1,69 & & 1,69 & 1,47 & 0,22 \\
Dic-16 & 1,47 & 1,55 & 1,60 & 1,69 & & 1,69 & 1,47 & 0,22 \\
Dic-17 & 1,47 & 1,55 & 1,60 & 1,69 & & 1,69 & 1,47 & 0,22 \\
\hline
\end{tabular}

Prima de Seguros

(\% Remuneración Bruta)

\begin{tabular}{r|ccccc|ccc}
\hline & Habitat & Integra & Prima & Profuturo & Horizonte & Max & Min & Rango \\
\hline Dic-08 & & 0,88 & 0,87 & 0,90 & 0,88 & 0,90 & 0,87 & 0,03 \\
Dic-09 & & 0,95 & 0,87 & 1,05 & 0,95 & 1,05 & 0,87 & 0,18 \\
Dic-10 & & 1,03 & 1,06 & 1,26 & 1,11 & 1,26 & 1,03 & 0,23 \\
Dic-11 & & 1,42 & 1,09 & 1,39 & 1,55 & 1,55 & 1,09 & 0,46 \\
Dic-12 & & 1,16 & 1,29 & 1,42 & 1,36 & 1,42 & 1,16 & 0,26 \\
Dic-13 & 1,23 & 1,23 & 1,23 & 1,23 & & 1,23 & 1,23 & 0,00 \\
Dic-14 & 1,23 & 1,23 & 1,23 & 1,23 & & 1,23 & 1,23 & 0,00 \\
Dic-15 & 1,33 & 1,33 & 1,33 & 1,33 & & 1,33 & 1,33 & 0,00 \\
Dic-16 & 1,33 & 1,33 & 1,33 & 1,33 & & 1,33 & 1,33 & 0,00 \\
Dic-17 & 1,36 & 1,36 & 1,36 & 1,36 & & 1,36 & 1,36 & 0,00 \\
\hline
\end{tabular}


Comisión Sobre el Flujo Total

(\% Remuneración Bruta)

\begin{tabular}{r|ccccc|ccc}
\hline & Habitat & Integra & Prima & Profuturo & Horizonte & Max & Min & Rango \\
\hline Dic-08 & & 2,68 & 2,62 & 2,88 & 2,83 & 2,88 & 2,62 & 0,26 \\
Dic-09 & & 2,75 & 2,62 & 3,35 & 2,90 & 3,35 & 2,62 & 0,73 \\
Dic-10 & & 2,83 & 2,81 & 3,56 & 3,06 & 3,56 & 2,81 & 0,75 \\
Dic-11 & & 3,22 & 2,84 & 3,56 & 3,50 & 3,56 & 2,84 & 0,72 \\
Dic-12 & & 2,90 & 2,89 & 3,52 & 3,25 & 3,52 & 2,89 & 0,63 \\
Dic-13 & 2,70 & 2,78 & 2,83 & 3,07 & & 3,07 & 2,70 & 0,37 \\
Dic-14 & 2,70 & 2,78 & 2,83 & 2,92 & & 2,92 & 2,70 & 0,22 \\
Dic-15 & 2,80 & 2,88 & 2,93 & 3,02 & & 3,02 & 2,80 & 0,22 \\
Dic-16 & 2,80 & 2,88 & 2,93 & 3,02 & & 3,02 & 2,80 & 0,22 \\
Dic-17 & 2,83 & 2,91 & 2,96 & 3,05 & & 3,05 & 2,83 & 0,22 \\
\hline Fuente: SBS
\end{tabular}

Fuente: SBS.

Elaboración: Propia.

Tabla 3.

Comisión Mixta

Comisión Sobre el Flujo (\% Remuneración Bruta)

\begin{tabular}{ccccc|ccc}
\hline & Habitat & Integra & Prima & Profuturo & Max & Min & Rango \\
\hline Dic-13 & 0,47 & 1,45 & 1,51 & 1,49 & 1,51 & 0,47 & 1,04 \\
Dic-14 & 0,47 & 1,45 & 1,51 & 1,49 & 1,51 & 0,47 & 1,04 \\
Dic-15 & 0,38 & 1,23 & 1,19 & 1,46 & 1,46 & 0,38 & 1,08 \\
Dic-16 & 0,38 & 1,23 & 1,19 & 1,46 & 1,46 & 0,38 & 1,08 \\
Dic-17 & 0,38 & 0,90 & 0,18 & 1,07 & 1,07 & 0,18 & 0,89 \\
\hline
\end{tabular}

Comisión Anual Sobre el Saldo (\%)

\begin{tabular}{ccccc|ccc}
\hline & Habitat & Integra & Prima & Profuturo & Max & Min & Rango \\
\hline Dic-13 & 1,25 & 1,20 & 1,25 & 1,20 & 1,25 & 1,20 & 0,05 \\
Dic-14 & 1,25 & 1,20 & 1,25 & 1,20 & 1,25 & 1,20 & 0,05 \\
Dic-15 & 1,25 & 1,20 & 1,25 & 1,20 & 1,25 & 1,20 & 0,05 \\
Dic-16 & 1,25 & 1,20 & 1,25 & 1,20 & 1,25 & 1,20 & 0,05 \\
Dic-17 & 1,25 & 1,20 & 1,25 & 1,20 & 1,25 & 1,20 & 0,05 \\
\hline
\end{tabular}

Prima de Seguros (\% Remuneración Bruta)

\begin{tabular}{ccccc|ccc}
\hline & Habitat & Integra & Prima & Profuturo & Max & Min & Rango \\
\hline Dic-13 & 1,23 & 1,23 & 1,23 & 1,23 & 1,23 & 1,23 & 0,00 \\
Dic-14 & 1,23 & 1,23 & 1,23 & 1,23 & 1,23 & 1,23 & 0,00 \\
Dic-15 & 1,33 & 1,33 & 1,33 & 1,33 & 1,33 & 1,33 & 0,00 \\
Dic-16 & 1,33 & 1,33 & 1,33 & 1,33 & 1,33 & 1,33 & 0,00 \\
Dic-17 & 1,36 & 1,36 & 1,36 & 1,36 & 1,36 & 1,36 & 0,00 \\
\hline Fuen
\end{tabular}

Fuente: SBS.

Elaboración: Propia.

De manera similar, en la Tabla 2 se muestra los niveles de la comisión mixta. Como indica la SBS, la comisión mixta se empieza a cobrar a partir del mes de febrero del año 2013 como parte de la implementación de la Ley $N^{\circ} 29903$,
Ley de la Reforma del SPP. Al igual que en el caso anterior, si observamos el componente sobre el flujo, destaca la similitud de evolución, aunque en niveles el rango para fines de 2017 es ligeramente mayor que en el caso anterior, 
$0.89 \%$, lo que resulta en un ahorro anual de S/ 118.37 aproximadamente para un afiliado cuyo salario es el mínimo de S/ 950, el cual también es un monto insignificante para incentivar al afiliado cambiarse de una AFP a otra. La comisión anual sobre el saldo presenta un panorama más drástico, dado que no hay mayor por AFP desde que se implementó este esquema, manteniendo el rango en $0.05 \%$, el cual no genera mayor incentivo para aquellos afiliados con un fondo de pensión basado en un salario mínimo. Finalmente, el componente del seguro es el mismo que el primer tipo de comisión analizado, por lo que no hay ahorro posible por ese concepto dado que las cuatro AFP cobran la misma prima de seguro, la cual además se ha ido incrementando en los últimos años.

A diferencia de la rentabilidad, las comisiones son variables determinísticas, es decir, su valor se sabe con anticipación, salvo que las AFP realicen cambios con mucha frecuencia, lo cual no suele ocurrir. Por lo tanto, los afiliados podrían usarla como una variable de decisión, pero dado que son similares entre las cuatro AFP, la decisión de pasarse de una a otra debido a las comisiones no tiene mucho impacto en la liquidez de los afiliados. De esta manera, al igual que la rentabilidad de los fondos de pensión, las comisiones pierden fuerza para ser una variable de decisión de los afiliados para escoger entre una AFP u otra, lo cual banaliza la existencia de cuatro AFP en el mercado.

\section{Otras variables de elección del SPP}

Otras variables que los afiliados podrían tener en cuenta son los servicios de pensión de jubilación, de pensión de sobrevivencia, pensión de invalidez o gastos en sepelio. Todos ellos no son observables por los afiliados hasta que esté próximo a jubilarse. Adicionalmente, dichos montos dependen directamente del valor del fondo que tenga y del marco legal vigente al momento de jubilarse. Por lo tanto, no son consideradas como tal en un mercado en el que, supuestamente, los afiliados deben escoger aquella que le brinda mejores servicios.

\section{RECOMENDACIONES DE POLÍTICA}

La coexistencia de dos sistemas de pensiones genera la pérdida de recursos para el Estado y para los afiliados. Por un lado, si bien el SPP ha cumplido un rol fundamental en el ámbito macroeconómico del país, desde el punto de vista del afiliado los resultados no son satisfactorios. Por otro lado, el SNP es un sistema que no es sostenible en el largo plazo, tal como lo demuestran los diversos estudios citados.

De esta manera, se propone una reforma del sistema de pensiones que consiste en crear una sola AFP y a la vez establecer un solo esquema de comisiones en función de los saldos con la finalidad de hacer auto sostenible esta nueva entidad, con lo cual se asignaría mejor los recursos y disminuiría la participación del Estado en los esquemas de disminuyendo el gasto del Estado en pensiones de jubilación.

a. Establecer una única APF pública y autónoma. Actualmente el SPP es un mercado oligopólico que no ofrece mayores beneficios a los afiliados debido a que brindan los mismos servicios a un alto costo. Por otro lado, el SNP no es sostenible en el largo plazo, principalmente porque compromete demasiado las cuentas fiscales lo cual pone en riesgo tanto el cumplimiento con las pensiones como el gasto público en otros temas relevantes como educación, salud o justicia. Por lo tanto, es más eficiente que una sola entidad se encargue de realizar la tarea de ambos sistemas ${ }^{3}$ y así aprovechar las economías de escala ${ }^{4}$. Tras 26 años de existencia del SPP, existe el conocimiento suficiente para que el sistema de pensiones sea administrado por una sola entidad. Esta entidad tendría que tener como base el mismo marco regulatorio del SPP, sobre todo lo relacionado al esquema de las cuentas individuales, supervisado por la SBS y el Banco Central de Reserva del Perú (BCRP). ${ }^{5}$

\footnotetext{
3 Los esquemas que quedarian en manos del Estado, a través de la ONP, son el resto de los esquemas pensionarios tal como el diseñado bajo la Ley $\mathrm{N}^{\circ} 20530$, los regímenes pensionarios del personal militar y policial de la Fuerza Armada y Fuerzas Policiales y la Caja de Beneficios Sociales del Pescador, quienes podrán ir incorporándose al nuevo sistema de manera voluntaria.

4 En la industria financiera las economias de escala se dan por el avance tecnológico y el capital humano especializado. En el caso de la administración de fondos, dado el volumen de afiliados y dada la tecnologia, el costo marginal de incorporar a un afiliado adicional es cercano a cero.

5 La Comisión de Protección Social (2017), creada por Resolución Ministerial $\mathrm{N}^{\circ}$ 017-2017-EF/10, propone "Un sistema integrado de pensiones que funcione sin discriminación alguna para todos los ciudadanos implica unir las fortalezas lortalezas (SPP) y programas de asistencia social Pensión 65), de forma (SPP) y programas de asistencia social Pensión 65), de forma
de consolidar sinergias que coadyuven a ofrecer pensiones de calidad superior." Dicho sistema lo denominan Centralizadora, la cual sería un tercer actor en el sistema de pensiones. Dicha propuesta no hace más que incorporar un tercer actor entre el SNP y el SPP, por lo que no se estaria ganando en eficiencia. Asimismo, la descripción de las funciones de dicha entidad entidad no es clara.
} 
Tabla 4

Estado de Resultados de las AFP (Millones de S/)

\begin{tabular}{|c|c|c|c|c|}
\hline & 2014 & 2015 & 2016 & 2017 \\
\hline 1. Ingresos & 1,143 & 1,199 & 1,236 & 1,223 \\
\hline 2. Gastos Operativos & 565 & 572 & 605 & 612 \\
\hline $\begin{array}{l}\text { 1.1 Gastos Administrativos } \\
\text { Del cual: }\end{array}$ & 383 & 402 & 436 & 450 \\
\hline Cargas de Personal & 147 & 153 & 159 & 164 \\
\hline Participaciones y Dietas del Directorio & 2 & 2 & 3 & 2 \\
\hline $\begin{array}{l}\text { 1.2 Gastos de Ventas } \\
\text { Del cual: }\end{array}$ & 182 & 170 & 169 & 162 \\
\hline Cargas de Personal & 125 & 116 & 114 & 118 \\
\hline Publicidad, Publicaciones y Relaciones Públicas & 28 & 26 & 24 & 18 \\
\hline 3. Utilidad Operativa & 578 & 627 & 631 & 610 \\
\hline 4. Otros Ingresos (Egresos) & 26 & 30 & 18 & 22 \\
\hline 5. Resultado Antes de Impuestos & 604 & 657 & 649 & 633 \\
\hline Impuesto a la Renta & -174 & -191 & -202 & -195 \\
\hline 6. Utilidad Neta & 429 & 466 & 448 & 438 \\
\hline
\end{tabular}

Pública, porque los afiliados deben tener acceso a sus servicios a costos reducidos, lo cual implica una disminución sustancial de las comisiones por la administración de los fondos. Disminuyendo las comisiones, se logrará atraer más afiliados de manera voluntaria en tanto estos perciban que obtienen buenos servicios a costos bajos.

Autónoma, porque sus políticas deben estar dirigidas exclusivamente a preservar el valor de los fondos administrados para la jubilación de sus afiliados, independientemente del Estado. Es crucial evitar la intervención del Estado en las políticas de inversión de la nueva entidad, ya que pueden estar motivados por consideraciones políticas antes que técnicas. La nueva entidad tiene que ser una netamente técnica, totalmente aislada de cuestiones políticas. Para ello, debe crearse el marco legal adecuado para lograr dicho objetivo.

Tal como se observa en la Tabla 4, para el año 2017 los ingresos por comisiones de las APF suman S/ 1,223 millones, con unos gastos operativos de $\mathrm{S} / 612$ millones y una utilidad neta de S/ 438 millones. La existencia de una única AFP sería viable con el actual nivel de ingresos, los cuales permitirían seguir cubriendo los gastos operativos, tanto del SPP como del SNP, considerando la reducción de costos por las economías de escala.

La existencia de una única AFP no sólo simplificaría el sistema nacional de pensiones, si no que reduciría sustancialmente la carga financiera del Estado para asignar recursos con fines de jubilación, debido a que la nueva entidad será auto sostenible en el largo plazo, tal como discutiremos en el siguiente punto.

Adicionalmente, al existir una sola AFP, quedaría margen para que las compañías de seguro compitan entre los afiliados para que estos elijan cuál de ellas le cobra menores primas $\mathrm{y}$ ofrece mejores rentas vitalicias al momento de jubilarse. En la actualidad, los afiliados no pueden elegir entre las compañías de seguro el momento que escogen en qué AFP aportar, sólo acepta la prima se seguro que le imponen.

b. Establecer una comisión como porcentaje del saldo del fondo de pensiones. La existencia de dos tipos de comisiones no 
es eficiente, porque los afiliados no saben exactamente cuáles son sus costos para compararlos. Por ese motivo, dado el tamaño actual del fondo de los afiliados es viable establecer una comisión en función del valor de los fondos y eliminar la comisión en función del salario bruto. Tal como se muestra en la Tabla 5, los ingresos representan el $0.79 \%$ del valor de los fondos, por lo que una primera propuesta de comisión sobre el saldo puede ser dicho porcentaje.

$\mathrm{Si}$ tomamos en cuenta que el objetivo principal de la nueva entidad debe ser preservar el valor de los fondos y no la acumulación de utilidades, dicha comisión debería estar cercana a $0.40 \%$, que representa el porcentaje de los Gastos Operativos sobre el Valor del Fondo de Pensiones. No obstante, dicho porcentaje incluye los Gastos de Ventas, por lo que, excluyéndolas de los Gastos Operativos, la comisión debería estar en un nivel, como mínimo, de $0.29 \%$, el cual representa los Gastos Administrativos como porcentaje del Valor del Fondo de Pensiones. Por lo tanto, la comisión sobre el saldo debe estar en el rango entre $0.79 \%$ y $0.29 \%$. En dicho rango debe estar incluido la prima de seguro, la cual puede ser elegida por el afiliado de acuerdo a la compañía de seguros que elija.

Tabla 5.

Estado de Resultados de las AFP

(\% del Valor de los Fondos de Pensiones)

\begin{tabular}{lcccc}
\hline & $\mathbf{2 0 1 4}$ & $\mathbf{2 0 1 5}$ & $\mathbf{2 0 1 6}$ & $\mathbf{2 0 1 7}$ \\
\hline Valor del Fondo de Pensiones (Mill. S/) & & & & \\
& $\mathbf{1 1 3 , 4 6 7}$ & $\mathbf{1 2 3 , 0 0 6}$ & $\mathbf{1 3 5 , 1 5 8}$ & $\mathbf{1 5 4 , 8 8 7}$ \\
1. Ingresos & $\mathbf{1 . 0 1}$ & $\mathbf{0 . 9 7}$ & $\mathbf{0 . 9 1}$ & $\mathbf{0 . 7 9}$ \\
2. Gastos Operativos & $\mathbf{0 . 5 0}$ & $\mathbf{0 . 4 7}$ & $\mathbf{0 . 4 5}$ & $\mathbf{0 . 4 0}$ \\
$\quad$ 1.1 Gastos Administrativos & 0.34 & 0.33 & 0.32 & 0.29 \\
$\quad$ Del cual: & & & & \\
$\quad$ Cargas de Personal & 0.13 & 0.12 & 0.12 & 0.11 \\
$\quad$ Participaciones y Dietas del Directorio & 0.00 & 0.00 & 0.00 & 0.00 \\
1.2 Gastos de Ventas & 0.16 & 0.14 & 0.13 & 0.10 \\
$\quad$ Del cual: & & & & \\
$\quad$ Cargas de Personal & 0.11 & 0.09 & 0.08 & 0.08 \\
$\quad$ Publicidad, Publicaciones y Relaciones Públicas & 0.02 & 0.02 & 0.02 & 0.01 \\
3. Utilidad Operativa & 0.51 & 0.51 & 0.47 & 0.39 \\
4. Otros Ingresos (Egresos) & 0.02 & 0.02 & 0.01 & 0.01 \\
5. Resultado Antes de Impuestos & 0.53 & 0.53 & 0.48 & 0.41 \\
$\quad$ Impuesto a la Renta & -0.15 & -0.16 & -0.15 & -0.13 \\
6. Utilidad Neta & 0.38 & 0.38 & 0.33 & 0.28 \\
$\quad$
\end{tabular}

Fuente: SBS. Boletín Mensual.

Elaboración: Propia.

Es importante mencionar que dichos ratios no consideran dos aspectos. Uno, son las economías de escala, por lo que el ratio debería ser menor, al ir reduciendo y mejorando procesos en la administración de fondos. Dos, al incorporar los fondos del SNP en la nueva entidad, el monto recaudado con los ratios propuestos sería mayor, por lo que tendría que disminuir por esa razón también. De esta manera, la comisión por saldo sería sustancialmente menor al $1.23 \%$ en promedio actualmente.

Por otro lado, uno de los cuestionamientos de los afiliados hacia el actual SPP es que las AFP siguen cobrando comisiones cuando hay 
eventos adversos que provocan la caída de la rentabilidad de los fondos, haciendo percibir al SPP como un sistema injusto desde el punto de vista de compartir los riesgos entre los afiliados y las AFP, dado que los afiliados están obligados a afiliarse a una AFP, mientras que estas ingresan voluntariamente al mercado.

En la Figura 1 se muestra la evolución de la rentabilidad de los tres distintos fondos de pensión y de la rentabilidad sobre los activos (ROA) de las AFP. Los periodos en los que hay discrepancia entre estas variables son 2008 y 2011. En 2008, mientras que la rentabilidad de los fondos 1,2 y 3 caían $4.26 \%, 21.87 \%$ y $37.84 \%$, el ROA de las AFP fue positivo, $0.40 \%$. La caída de la rentabilidad de los fondos de pensión se explicó por la crisis financiera en los Estados Unidos de América. Por otro lado, en 2011, se observó un panorama similar, registrándose rentabilidades de $3.02 \%,-7.06 \%$ y $-17.32 \%$ en los fondos respectivos, mientras que el ROA del SPP fue $18.25 \%$. La caída de los fondos se debido a la crisis soberana en Europa la cual generó el temor de que se transmitiera a las economías en desarrollo.

Figura 1

Rentabilidad de los Fondos versus de las AFP (\%)

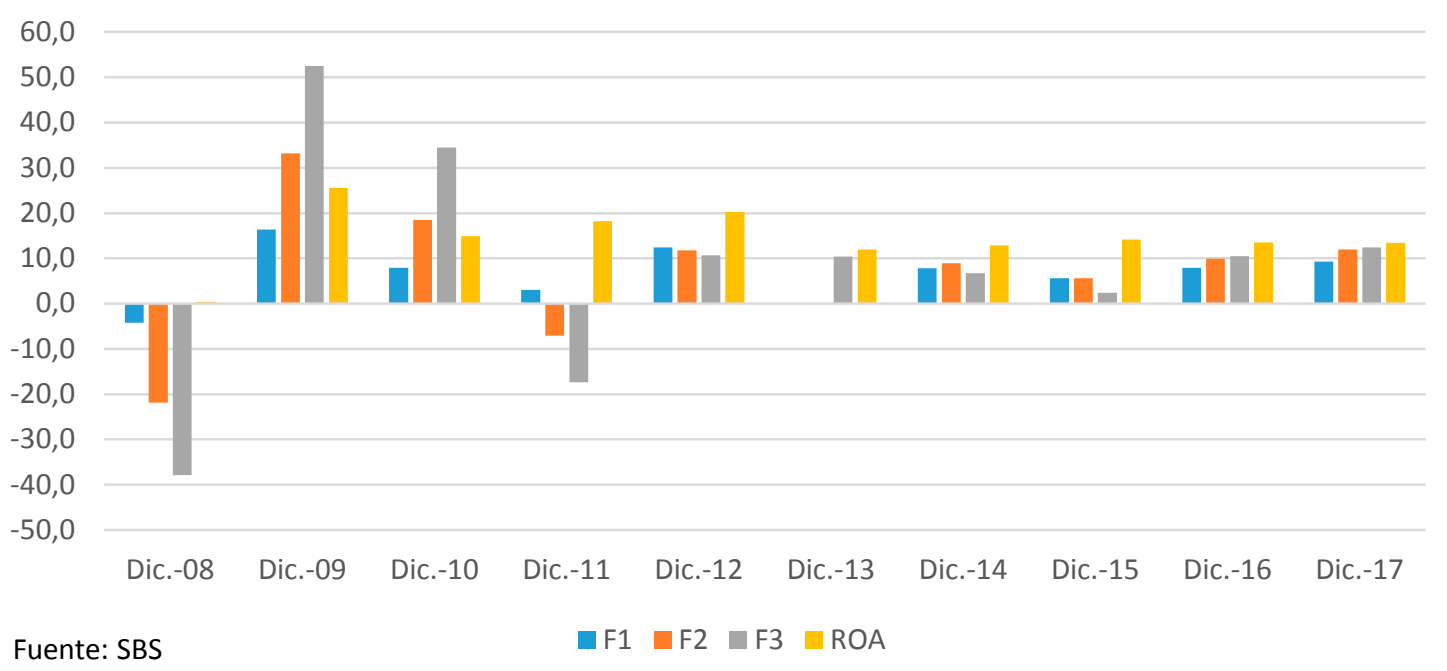

Lo anterior no llamaría la atención si la participación de los afiliados fuera voluntaria, por lo que supuestamente deberían entender que están expuestos a pérdida de capital, lo cual implica que el pago de comisiones se mantiene a pesar de los malos resultados, como en el caso de la crisis financiera de los EE.UU. durante 2007 - 2009 tal como lo indica Prager (2012), en donde los intermediarios cobraron grandes sumas de dinero a pesar de las pérdidas generadas por sus gestiones. Esto disminuye la percepción de bienestar de los afiliados con respecto al SPP, debido a que, ante eventos adversos del mercado, los únicos perdedores son ellos $\mathrm{y}$ no los administradores de los fondos. Como se discute en la teoría del bienestar, esta no es absoluta, si no relativa ${ }^{6}$ tal como lo indican Sen (1983) y Rablen (2008), por lo que estos eventos no hacen más que mermar la sostenibilidad del actual SPP.

Así, la nueva comisión tiene que estar en función de la rentabilidad de los fondos, es decir, mientras la rentabilidad de los fondos sea negativa, entonces la entidad no debe cobrar comisiones, logrando no sólo disminuir las pérdidas de los afiliados, si no también otorgando credibilidad la nueva

\footnotetext{
6 "The relativist approach sees deprivation in terms of a person or a household being able to achieve less than what others in that society do [...]" Sen (1983).
} 
entidad al observar que las pérdidas, debido a la evolución del mercado, es compartida tanto por los afiliados como por la entidad administradora de fondos. No obstante, esto no debe comprometer la continuidad de la administración de los fondos, para lo cual es necesario que la entidad realice provisiones durante los periodos de bonanza con la finalidad de que cubra sus gastos operativos cuando no pueda cobrar comisiones. Por otro lado, es indispensable que tanto la SBS y el BCRP, como supervisores de esta nueva entidad, construyan índices de referencia de rentabilidad de los fondos para que monitoreen y elaboren recomendaciones con respecto a la administración de los fondos de los afiliados.

Al establecerse una comisión única en función del saldo, con las particularidades señaladas, se eliminaría el costo del 3\% del salario bruto de los trabajadores, descontándoles sólo el $10 \%$. El aporte del $10 \%$ de salario bruto es suficiente, debido a que dicho descuento es como un impuesto adicional para los trabajadores que les resta liquidez en el presente, por lo que tratar de incrementarlo con el objetivo de mejorar las pensiones de los afiliados no es viable. Queda de lado de la nueva entidad establecer las políticas para los casos en que no se logre cubrir una pensión mínima.

\section{CONCLUSIONES}

En este artículo se propone una reforma del sistema de pensiones en el Perú que consiste crear una Administradora de Fondos de Pensiones, pública y autónoma, de tal manera que absorba la tarea que realizan el SNP, bajo la Ley $\mathrm{N}^{\circ} 19990$, y el SPP, ello con la finalidad de ganar mayor eficiencia en la asignación de recursos de los afiliados y del Estado. Asimismo, se plantea el diseño de una comisión por la administración de los fondos de pensión única en función al saldo de los fondos de pensión y de la rentabilidad de estos, a través de la cual hará sostenible la labor de administración de los fondos de pensiones.

El sistema de pensiones en el Perú actualmente se compone por dos sistemas: el SNP y el SPP. El primero ha sido analizado extensamente y las conclusiones confluyen en mostrar la insostenibilidad financiera en el largo plazo. Si bien el segundo también ha sido analizado con similar rigurosidad, los estudios no concluyen sobre su insostenibilidad financiera, pero si sobre varios aspectos que deben ser mejorados. En ese sentido, a través del análisis del SPP desde el punto de vista del afiliado en dicho mercado, se muestra que el esquema oligopólico de dicho sistema no contribuye al bienestar del afiliado. Así, por un lado, el SNP es financieramente insostenible en el largo plazo, y por otro lado el SPP no aporta mayor bienestar a los afiliados que están en dicho mercado, por lo tanto, se propone la idea esgrimida en el párrafo anterior.

El debate para lograr un sistema de pensiones ideal es permanente en toda sociedad y en todo tiempo. En ese sentido, este artículo contribuye al mismo, proponiendo la creación de una única AFP con carácter público y autónomo, con la finalidad de otorgarle como objetivo único preservar el valor de los fondos de pensiones y no la acumulación de utilidades, y con el objetivo de aislarla de toda injerencia política. Asimismo, se propone la implementación de una comisión única en función del saldo de los fondos de pensiones y de su rentabilidad. De esta manera, se puede disminuir la mala asignación de recursos de los afiliados y del Estado.

En este artículo no se discute sobre cómo ampliar la cobertura o el nivel de pensiones. Partiendo de una entidad que administre de manera adecuada los fondos de pensión a bajos costos, se puede replantear la discusión sobre cómo incrementar la cobertura o el nivel de pensiones de jubilados.

\section{REFERENCIAS}

Alonso, J., Sánchez, R., y D. Tuesta (2014) "Un modelo para el sistema de pensiones en el Perú: Diagnóstico y recomendaciones", Revista Estudios Económicos, Banco Central de Reserva del Perú, junio, Pp. 81-98.

Carranza, L., Melguizo, A. y D. Tuesta (Ed.) (2016) Ideas para una Reforma de Pensiones. Lima, Perú: Universidad de San Martín de Porres, 342 pp.

Comisión de Protección Social (2017) "Propuestas de Reforma en el Sistema de Pensiones, Financiamiento en la Salud y Seguro de Desempleo", URL http://www. proteccionsocial.com.pe/

Cruz-Saco, M., Mendoza, J., y B. Seminario (2014) "El Sistema Previsional del Perú: Diagnóstico 1996-2013, Proyecciones 20142050 y Reforma", Documento de Discusión DD/14/11, Universidad el Pacífico, 41 pp. 
International Monetary Fund (IMF) (2005) "Ageing and pension system reform: implications for financial markets and economic policies", 77 pp.

Mendiola, A., Aguirre, C., Buendía, D., Chong Shing, J., Segura, M. y M. Segura (2013) "Análisis del sistema privado de pensiones: propuesta de reforma y generación de valor", Serie Gerencia para el Desarrollo, Universidad ESAN, No 29, 164 pp.

Ministerio de Economía y Finanzas, MEF (2004) "Informe Trimestral: Los Sistemas de Pensiones en el Perú", Dirección General de Asuntos Económicos y Sociales, 75 pp.

Muñoz, Ítalo (2000) “La reforma del sistema privado de pensiones" En: Abusada, R., Du Bois, F., Morón, E., y J. Valderrama (2000) La Reforma Incompleta. Rescatando los Noventa. Lima, Perú: Universidad del Pacífico, 473 Pp.
Prager, Jonas (2012) "The Financial Crisis of 2007/8: Misaligned Incentives, Bank Mismanagement, and Troubling Policy Implications", Department of Economics New York University, 44 pp.

Rablen, Matthew D. (2008) "Relativity, Rank and the Utility of Income", The Economic Journal, Vol. 118, No. 528, pp. 801-821.

Sen, Amartya (1983) "Poor, Relatively Speaking", Oxford Economic Papers, New Series, Vol. 35, No. 2, pp. 153-169. 